\title{
A Contraction Fixed Point Theorem in Partially Ordered Metric Spaces and Application to Fractional Differential Equations
}

\author{
Xiangbing Zhou, Wenquan Wu, and Hongjiang Ma \\ Department of Computer Science, Aba Teachers College, Sichuan, Wenchuan 623002, China \\ Correspondence should be addressed to Xiangbing Zhou, studydear@gmail.com \\ Received 23 September 2012; Accepted 31 October 2012 \\ Academic Editor: Xinguang Zhang
}

Copyright (c) 2012 Xiangbing Zhou et al. This is an open access article distributed under the Creative Commons Attribution License, which permits unrestricted use, distribution, and reproduction in any medium, provided the original work is properly cited.

We generalize a fixed point theorem in partially ordered complete metric spaces in the study of A. Amini-Harandi and H. Emami (2010). We also give an application on the existence and uniqueness of the positive solution of a multipoint boundary value problem with fractional derivatives.

\section{Introduction}

Let $\mathcal{S}$ denote the class of those functions $\beta:[0,+\infty) \rightarrow[0,1)$ which satisfies the condition

$$
\beta\left(t_{n}\right) \longrightarrow 1 \text { implies } t_{n} \longrightarrow 0
$$

The following generalization of Banach's contraction principle is due to Geraghty [1].

Theorem 1.1. Let $(M, d)$ be a complete metric space and let $f: M \rightarrow M$ be a map. Suppose there exists $\beta \in \mathcal{S}$ such that for each $x, y \in M$,

$$
d(f(x), f(y)) \leq \beta(d(x, y)) d(x, y) .
$$

Then $f$ has a unique fixed point $z \in M$, and $\left\{f^{n}(x)\right\}$ converges to $z$, for each $x \in M$.

And then, Amini-Harandi and Emami [2] proved a version of Theorem 1.1 in the context of partially ordered complete metric spaces. 
Theorem 1.2. Let $(M, \preceq)$ be a partially ordered set and suppose that there exists a metric $d$ in $M$ such that $(M, d)$ is a complete metric space. Let $f: M \rightarrow M$ be a nondecreasing mapping, and there exists an element $x_{0} \in M$ with $x_{0} \leq f\left(x_{0}\right)$. Suppose that there exists $\beta \in \mathcal{S}$ such that

$$
d(f(x), f(y)) \leq \beta(d(x, y)) d(x, y), \quad \text { for each } x, y \in M \text { with } x \geq y \text {. }
$$

Assume that either $f$ is continuous or $M$ is such that

$$
\text { if an increasing sequence }\left\{x_{n}\right\} \longrightarrow x \text { in } M \text {, then } x_{n} \leq x, \forall n \text {. }
$$

Besides, if

$$
\text { for any } x, y \in M \text {, there exists } z \in M \text { which is comparable to } x \text { and } y \text {, }
$$

Then $f$ has a unique fixed point.

Theorem 2.2 was also applied to obtain the existence and uniqueness of the solution of a periodic boundary value problem by Amini-Harandi and Emami [2] and a singular fractional three-point boundary value problem by Cabrera et al. [3].

\section{Main Results}

In this section, we firstly define a class of functions $\mathcal{A}$ by $\beta:[0,+\infty) \rightarrow[0,+\infty)$, and there exists a constant $K>0$ such that

$$
\sup _{t \in[0,+\infty)}\{\beta(t)\} \leq K
$$

Remark 2.1. Function classes $\mathcal{A}$ include all bounded functions on $[0,+\infty)$ with upper bound $K$ which are more extensive than those of $\mathcal{S}$. For example, $\beta(t)=K \sin t \in \mathcal{A}$ but not in $\mathcal{S}$.

Now, we give an extended version of Theorem 1.2 in the context of partially ordered complete metric spaces.

Theorem 2.2. Let $(M, \preceq)$ be a partially ordered set and suppose that there exists a metric $d$ in $M$ such that $(M, d)$ is a complete metric space. Let $f: M \rightarrow M$ be a nondecreasing mapping, and there exists s an element $x_{0} \in M$ with $x_{0} \leq f\left(x_{0}\right)$. Suppose that there exists a constant $\theta \in(0,1 / K)$ and $\beta \in \mathcal{S}$ such that

$$
d(f(x), f(y)) \leq \theta \beta(\theta d(x, y)) d(x, y), \quad \text { for each } x, y \in M \text { with } x \geq y \text {. }
$$

Assume that either $f$ is continuous or

$$
\text { if an increasing sequence }\left\{x_{n}\right\} \longrightarrow x \text { in } M \text {, then } x_{n} \preceq x, \forall n \text {. }
$$


Besides, if

for any $x, y \in M$, there exists $z \in M$ which is comparable to $x$ and $y$,

then $f$ has a unique fixed point.

Proof. We first show that $f$ has a fixed point. Since $x_{0} \leq f\left(x_{0}\right)$ and $f$ is an increasing function, we obtain by induction that

$$
x_{0} \preceq f\left(x_{0}\right) \preceq f^{2}\left(x_{0}\right) \preceq f^{3}\left(x_{0}\right) \preceq \cdots \preceq f^{n}\left(x_{0}\right) \preceq \cdots .
$$

Put $x_{n+1}=f^{n}\left(x_{0}\right), n=1,2, \ldots$ For each integer $n \geq 1$, from (2.5), we have $x_{n} \preceq x_{n+1}$, then by (2.2)

$$
d\left(x_{n+1}, x_{n}\right)=d\left(f^{n}\left(x_{0}\right), f^{n-1}\left(x_{0}\right)\right) \leq \theta \beta\left(\theta d\left(x_{n}, x_{n-1}\right)\right) d\left(x_{n}, x_{n-1}\right) \leq K \theta d\left(x_{n}, x_{n-1}\right) .
$$

If there exists $n_{0} \in \mathbb{N}$ such that $d\left(x_{n_{0}}, x_{n_{0}-1}\right)=0$, then $x_{n_{0}}=f^{n_{0}-1}\left(x_{0}\right)=f\left(x_{n_{0}-1}\right)=x_{n_{0}-1}$ and $x_{n_{0}-1}$ is a fixed point; in this case, the proof is finished. Otherwise, for any $n \in \mathbb{N}$, $d\left(x_{n}, x_{n-1}\right) \neq 0$. Then by (2.6), we have

$$
d\left(x_{n+1}, x_{n}\right) \leq K \theta d\left(x_{n}, x_{n-1}\right) \leq \cdots \leq(K \theta)^{n+1} d\left(x_{n_{0}}, x_{n_{0}-1}\right) \longrightarrow 0, \quad \text { as } n \longrightarrow+\infty,
$$

that is,

$$
d\left(x_{n+1}, x_{n}\right) \longrightarrow 0, \quad \text { as } n \longrightarrow+\infty
$$

Now, we show that $x_{n}$ is a Cauchy sequence. By the triangle inequality and (2.2), we have

$$
\begin{aligned}
d\left(x_{n}, x_{m}\right) & \leq d\left(x_{n}, x_{n+1}\right)+d\left(x_{n+1}, x_{m+1}\right)+d\left(x_{m+1}, x_{m}\right) \\
& \leq d\left(x_{n}, x_{n+1}\right)+\theta \beta\left(\theta d\left(x_{n}, x_{m}\right)\right) d\left(x_{n}, x_{m}\right)+d\left(x_{m+1}, x_{m}\right) \\
& \leq d\left(x_{n}, x_{n+1}\right)+\theta K d\left(x_{n}, x_{m}\right)+d\left(x_{m+1}, x_{m}\right),
\end{aligned}
$$

and then

$$
d\left(x_{n}, x_{m}\right) \leq(1-K \theta)^{-1}\left[d\left(x_{n}, x_{n+1}\right)+d\left(x_{m+1}, x_{m}\right)\right] \longrightarrow 0, \quad \text { as } m, n \longrightarrow+\infty,
$$

which implies that $x_{n}$ is a Cauchy sequence in $M$. Since $(M, d)$ is a complete metric space, then there exists a $z \in M$ such that $\lim _{n \rightarrow+\infty} x_{n}=z$. To prove that $z$ is a fixed point of $f$, if $f$ is continuous, then

$$
z=\lim _{n \rightarrow+\infty} x_{n}=\lim _{n \rightarrow+\infty} f^{n}\left(x_{0}\right)=\lim _{n \rightarrow+\infty} f^{n+1}\left(x_{0}\right)=f\left(\lim _{n \rightarrow+\infty} f^{n}\left(x_{0}\right)\right)=f(z),
$$


hence $z=f(z)$. If case (2.3) holds, then we claim that $f(z)=z$ still holds. In fact,

$$
d(f(z), z) \leq d\left(f(z), f\left(x_{n}\right)\right)+d\left(f\left(x_{n}\right), z\right) \leq \theta K d\left(z, x_{n}\right)+d\left(x_{n+1}, z\right)
$$

since $d\left(z, x_{n}\right) \rightarrow 0$, taking limit as $n \rightarrow+\infty$, then $d(f(z), z) \leq 0$, this proves that $d(f(z), z)=$ 0 ; consequently, $f(z)=z$.

Let $y$ be another fixed point of $f$. From (2.4) there exists $x \in M$ which is comparable to $y$ and $z$. Monotonicity implies that $f^{n}(x)$ is comparable to $f^{n}(y)=y$ and $f^{n}(z)=z$ for $n=0,1,2 \ldots$ Moreover,

$$
d\left(z, f^{n}(x)\right)=d\left(f^{n}(z), f^{n}(x)\right) \leq K \theta d\left(z, f^{n-1}(x)\right) .
$$

Taking limit, and then $\lim _{n \rightarrow+\infty} d\left(z, f^{n}(x)\right)=0$. Similar to [2], we have $d(z, y)=0$. The proof of the uniqueness of the fixed point is completed.

\section{Application to Fractional Differential Equations}

In this section, we consider the unique positive solution for a general higher order fractional differential equation by using the generalized fixed point theorem

$$
\begin{array}{r}
-\boldsymbol{\Phi}^{\alpha} x(t)=f\left(t, x(t), \boldsymbol{\Phi}^{\mu_{1}} x(t), \boldsymbol{\Phi}^{\mu_{2}} x(t), \ldots, \boldsymbol{\Phi}^{\mu_{n-1}} x(t)\right), \\
\boldsymbol{\Phi}^{\mu_{i}} x(0)=0, \quad 1 \leq i \leq n-1, \\
\boldsymbol{刃}^{\mu_{n-1}+1} x(0)=0, \quad \boldsymbol{刃}^{\mu_{n-1}} x(1)=\sum_{j=1}^{m-2} a_{j} \boldsymbol{\Phi}^{\mu_{n-1}} x\left(\xi_{j}\right),
\end{array}
$$

where $n-1<\alpha \leq n, n \in \mathbb{N}$ and $n \geq 3$ with $0<\mu_{1}<\mu_{2}<\cdots<\mu_{n-2}<\mu_{n-1}$ and $n-3<\mu_{n-1}<$ $\alpha-2, a_{j} \in \mathbb{R}, 0<\xi_{1}<\xi_{2}<\cdots<\xi_{m-2}<1$ satisfying $0<\sum_{j=1}^{m-2} a_{j} \xi_{j}^{\alpha-\mu_{n-1}-1}<1$ and $\boldsymbol{\Phi}^{\alpha}$ is the standard Riemann-Liouville derivative, $f \in C\left([0,1] \times \mathbb{R}^{n},[0,+\infty)\right)$. Recently, there has been a significant development in the study of fractional differential equations; for more details we refer the reader to [4-12] and the references cited therein.

For the convenience of the reader, we present some notations and lemmas which will be used in the proof of our results.

Definition 3.1 (see $[13,14]$ ). The Riemann-Liouville fractional integral of order $\alpha>0$ of a function $x:(0,+\infty) \rightarrow \mathbb{R}$ is given by

$$
I^{\alpha} x(t)=\frac{1}{\Gamma(\alpha)} \int_{0}^{t}(t-s)^{\alpha-1} x(s) d s,
$$

provided that the right-hand side is pointwise defined on $(0,+\infty)$. 
Definition 3.2 (see $[13,14])$. The Riemann-Liouville fractional derivative of order $\alpha>0$ of a function $x:(0,+\infty) \rightarrow \mathbb{R}$ is given by

$$
\boldsymbol{\Phi}_{\mathrm{t}}^{\alpha} x(t)=\frac{1}{\Gamma(n-\alpha)}\left(\frac{d}{d t}\right)^{n} \int_{0}^{t}(t-s)^{n-\alpha-1} x(s) d s,
$$

where $n=[\alpha]+1,[\alpha]$ denotes the integer part of number $\alpha$, provided that the right-hand side is pointwise defined on $(0,+\infty)$.

Proposition 3.3 (see $[13,14])$. Consider the following.

(1) If $x \in L^{1}(0,1), v>\sigma>0$, then

$$
I^{v} I^{\sigma} x(t)=I^{v+\sigma} x(t), \quad \boldsymbol{\Phi}_{\mathrm{t}}^{\sigma} I^{v} x(t)=I^{v-\sigma} x(t), \quad \boldsymbol{\Phi}_{\mathrm{t}}^{\sigma} I^{\sigma} x(t)=x(t) .
$$

(2) If $v>0, \sigma>0$, then

$$
\boldsymbol{\Phi}_{\mathbf{t}}^{v} t^{\sigma-1}=\frac{\Gamma(\sigma)}{\Gamma(\sigma-v)} t^{\sigma-v-1}
$$

Proposition 3.4 (see $[13,14])$. Let $\alpha>0$, and $f(x)$ is integrable, then

$$
I^{\alpha} \boldsymbol{\Phi}_{\mathbf{t}}^{\alpha} x(t)=f(x)+c_{1} x^{\alpha-1}+c_{2} x^{\alpha-2}+\cdots+c_{n} x^{\alpha-n}
$$

where $c_{i} \in \mathbb{R}(i=1,2, \ldots, n), n$ is the smallest integer greater than or equal to $\alpha$.

Lemma 3.5 (see [15]). Let $x(t)=I^{\mu_{n-1}} u(t)$, then BVP (3.1) is equivalent to the following BVP:

$$
\begin{gathered}
-\boldsymbol{\Phi}^{\alpha-\mu_{n-1}} u(t)=f\left(t, I^{\mu_{n-1}} u(t), I^{\mu_{n-1}-\mu_{1}} u(t), \ldots, I^{\mu_{n-1}-\mu_{n-2}} u(t), u(t)\right), \\
u(0)=u^{\prime}(0)=0 \quad u(1)=\sum_{j=1}^{m-2} a_{j} u\left(\xi_{j}\right) .
\end{gathered}
$$

Moreover, if $v \in C([0,1],[0,+\infty))$ is a solution of problem (3.7), then the function $x(t)=I^{\mu_{n-1}} u(t)$ is a positive solution of problem (3.1).

Let

$$
k(t, s)= \begin{cases}\frac{(t(1-s))^{\alpha-\mu_{n-1}-1}-(t-s)^{\alpha-\mu_{n-1}-1}}{\Gamma\left(\alpha-\mu_{n-1}\right)}, & 0 \leq s \leq t \leq 1, \\ \frac{(t(1-s))^{\alpha-\mu_{n-1}-1}}{\Gamma\left(\alpha-\mu_{n-1}\right)}, & 0 \leq t \leq s \leq 1\end{cases}
$$


from [15], the Green function of (3.7) is

$$
H(t, s)=k(t, s)+\frac{t^{\alpha-\mu_{n-1}-1}}{1-\sum_{j=1}^{m-2} a_{j} \xi_{j}^{\alpha-\mu_{n-1}-1}} \sum_{j=1}^{m-2} a_{j} k\left(\xi_{j}, s\right)
$$

and with property

$$
0 \leq H(t, s) \leq \frac{1}{\Gamma\left(\alpha-\mu_{n-1}\right)}\left(1+\frac{\sum_{j=1}^{m-2} a_{j}}{1-\sum_{j=1}^{m-2} a_{j} \xi_{j}^{\alpha-\mu_{n-1}-1}}\right)=\eta .
$$

In our considerations, we will work in the Banach space $E=C([0,1] ; \mathbb{R})$ with the classical metric given by $d(x, y)=\sup _{0 \leq t \leq 1}|x(t)-y(t)|$. Notice that this space can be equipped with a partial order given by

$$
x, y \in C[0,1], \quad x \leq y \Longleftrightarrow x(t) \leq y(t) \text { for any } t \in[0,1]
$$

In [2], it is proved that $(C[0,1], \leq)$ satisfies condition (2.3) of Theorem 2.2. Moreover, for $x, y \in$ $C[0,1]$, as the function $\max \{x, y\} \in C[0,1],(C[0,1], \leq)$ satisfies condition [15]. Consider the cone

$$
P=\{u \in C[0,1]: u(t) \geq 0\} .
$$

Note that as $P$ is a closed set of $C[0,1], P$ is a complete metric space.

It is well known that the BVP (3.7) is equivalent to the integral equation

$$
u(t)=\int_{0}^{1} H(t, s) f\left(s, I^{\mu_{n-1}} u(s), I^{\mu_{n-1}-\mu_{1}} u(s), \ldots, I^{\mu_{n-1}-\mu_{n-2}} u(s), u(s)\right) d s
$$

Now, for $u \in P$ we define the operator $T$ by

$$
(T u)(t)=\int_{0}^{1} H(t, s) f\left(s, I^{\mu_{n-1}} u(s), I^{\mu_{n-1}-\mu_{1}} u(s), \ldots, I^{\mu_{n-1}-\mu_{n-2}} u(s), u(s)\right) d s .
$$

Then from the assumption on $f$ and (3.10), we have

$$
T(P) \subset P .
$$

We also introduce the following class of nondecreasing functions $B$ by $\phi:[0,+\infty) \rightarrow$ $[0,+\infty)$ satisfying the following:

$$
\phi(x) \leq K x, \text { for any } x>0
$$


Clearly, if $\phi \in \mathbb{B}$, then $\phi(x) / x \in \mathcal{A}$. The standard functions $\phi \in \mathbb{B}$, for example, $\phi(x)=K x$, $\phi(x)=(2 K / \pi) x \arctan x$, and $\phi(x)=K x^{2} /(1+x)$.

Theorem 3.6. Suppose $f\left(t, x_{1}, x_{2}, \ldots, x_{n}\right)$ is nondecreasing in $x_{i}$ on $[0,+\infty)$; moreover, there exist $n$ positive constants $\rho_{i}, i=1,2, \ldots, n$ that satisfy

$$
\max \left\{\rho_{1}, \rho_{2}, \ldots, \rho_{n}\right\} \leq(n \eta)^{-1}
$$

and there exist a function $\phi \in \mathbb{B}$ and constants $0<\theta_{1}<\Gamma\left(\mu_{n-1}\right) / K, 0<\theta_{n}<1 / K, 0<\theta_{i}<$ $\Gamma\left(\mu_{n-1}-\mu_{i}\right) / K, i=1,2, \ldots, n-2$ such that

$$
\left|f\left(t, x_{1}, x_{2}, \ldots, x_{n}\right)-f\left(t, y_{1}, y_{2}, \ldots, y_{n}\right)\right| \leq \sum_{i=1}^{n} \rho_{i} \phi\left(\theta_{i}\left(x_{i}-y_{i}\right)\right)
$$

for $x_{i}, y_{i} \in[0,+\infty), i=1,2, \ldots, n$ with $x_{i} \geq y_{i}$ and $t \in[0,1]$. Then problem (3.1) has a unique nonnegative solution.

Proof. We check that the hypotheses in Theorem 2.2 are satisfied.

Firstly, the operator $T$ is nondecreasing by the hypothesis. Then for any $u \geq v$, we have

$$
\begin{aligned}
(T u)(t) & =\int_{0}^{1} H(t, s) f\left(s, I^{\mu_{n-1}} u(s), I^{\mu_{n-1}-\mu_{1}} u(s), \ldots, I^{\mu_{n-1}-\mu_{n-2}} u(s), u(s)\right) d s \\
& \geq \int_{0}^{1} H(t, s) f\left(s, I^{\mu_{n-1}} v(s), I^{\mu_{n-1}-\mu_{1}} v(s), \ldots, I^{\mu_{n-1}-\mu_{n-2}} v(s), v(s)\right) d s \\
& =(T v)(t) .
\end{aligned}
$$

Noticing that

$$
\begin{gathered}
u(s)-v(s) \leq d(u, v) \\
I^{\mu_{n-1}} u(s)-I^{\mu_{n-1}} v(s) \leq \int_{0}^{t} \frac{(t-s)^{\mu_{n-1}-1}|u(s)-v(s)|}{\Gamma\left(\mu_{n-1}\right)} d s \leq \frac{d(u, v)}{\Gamma\left(\mu_{n-1}\right)} \\
I^{\mu_{n-1}-\mu_{i}} u(s)-I^{\mu_{n-1}-\mu_{i}} v(s) \leq \int_{0}^{t} \frac{(t-s)^{\mu_{n-1}-\mu_{i}-1}|u(s)-v(s)|}{\Gamma\left(\mu_{n-1}-\mu_{i}\right)} d s \\
\leq \frac{d(u, v)}{\Gamma\left(\mu_{n-1}-\mu_{i}\right)}, \quad i=1,2, \ldots, n-2,
\end{gathered}
$$

and take

$$
\theta=\max \left\{\frac{\theta_{1}}{\Gamma\left(\mu_{n-1}\right)}, \frac{\theta_{2}}{\Gamma\left(\mu_{n-1}-\mu_{1}\right)}, \ldots, \frac{\theta_{n-1}}{\Gamma\left(\mu_{n-1}-\mu_{n-2}\right)}, \theta_{n}\right\}
$$


Thus, for any $u \geq v$, we have

$$
\begin{aligned}
& d(T u, T v)= \max _{t \in[0,1]}|T u(t)-T v(t)| \\
& \leq \eta \int_{0}^{1} \mid f\left(s, I^{\mu_{n-1}} u(s), I^{\mu_{n-1}-\mu_{1}} u(s), \ldots, I^{\mu_{n-1}-\mu_{n-2}} u(s), u(s)\right) d s \\
& \quad \quad-f\left(s, I^{\mu_{n-1}} v(s), I^{\mu_{n-1}-\mu_{1}} v(s), \ldots, I^{\mu_{n-1}-\mu_{n-2}} v(s), v(s)\right) \mid \\
& \leq \eta \int_{0}^{1}\left[\rho_{1} \phi\left(\theta_{1}\left(I^{\mu_{n-1}} u(s)-I^{\mu_{n-1}} v(s)\right)\right)+\rho_{2} \phi\left(\theta_{2}\left(I^{\mu_{n-1}-\mu_{1}} u(s)-I^{\mu_{n-1}-\mu_{1}} v(s)\right)\right)\right. \\
&\left.\quad+\cdots+\rho_{n-1} \phi\left(\theta_{n-1}\left(I^{\mu_{n-1}-\mu_{n-2}} u(s)-I^{\mu_{n-1}-\mu_{n-2}} v(s)\right)\right)+\rho_{n} \phi\left(\theta_{n}(u(s)-v(s))\right)\right] d s \\
& \leq n \eta \max \left\{\rho_{1}, \rho_{2}, \ldots, \rho_{n}\right\} \phi(\theta d(u, v)) \leq \phi(\theta d(u, v)) .
\end{aligned}
$$

For $u \neq v$, we have

$$
d(T u, T v) \leq \frac{\phi(\theta d(u, v))}{\theta d(u, v)} \theta d(u, v)=\beta(\theta d(u, v)) \theta d(u, v)
$$

and this inequality is obviously satisfied for $u=v$. Thus, we have

$$
d(T u, T v) \leq \frac{\phi(\theta d(u, v))}{\theta d(u, v)} \theta d(u, v)=\beta(\theta d(u, v)) \theta d(u, v), \quad \text { for any } u, v \in P \text { with } u \geq v \text {. }
$$

Finally, since the zero function satisfies $0 \leq T 0$, Theorem 2.2 tells us that the operator $T$ has a unique fixed point in $P$, or, equivalently, the BVP (3.1) has a unique nonnegative solution $x$ in $C[0,1]$.

Theorem 3.7. If the assumptions of Theorem 3.6 are satisfied, and there exists $t_{0} \in[0,1]$ such that $f\left(t_{0}, 0, \ldots, 0\right) \neq 0$, then the unique solution of (3.1) is positive (a positive solution means a solution satisfying $x(t)>0$ for $t \in(0,1)$ ).

Proof. By Theorem 3.6, the problem (3.1) has a unique nonnegative solution. We prove the nonnegative solution is also positive.

Otherwise, there exists $0<t^{*}<1$ such that $x\left(t^{*}\right)=0$, and

$$
x\left(t^{*}\right)=\int_{0}^{1} H\left(t^{*}, s\right) f\left(s, I^{\mu_{n-1}} u(s), I^{\mu_{n-1}-\mu_{1}} u(s), \ldots, I^{\mu_{n-1}-\mu_{n-2}} u(s), u(s)\right) d s=0
$$


Then

$$
\begin{aligned}
0=x\left(t^{*}\right) & =\int_{0}^{1} H\left(t^{*}, s\right) f\left(s, I^{\mu_{n-1}} u(s), I^{\mu_{n-1}-\mu_{1}} u(s), \ldots, I^{\mu_{n-1}-\mu_{n-2}} u(s), u(s)\right) d s \\
& \geq \int_{0}^{1} H\left(t^{*}, s\right) f(s, 0, \ldots, 0) d s \geq 0 .
\end{aligned}
$$

Consequently,

$$
\int_{0}^{1} H\left(t^{*}, s\right) f(s, 0, \ldots, 0) d s=0
$$

this yields

$$
H\left(t^{*}, s\right) f(s, 0, \ldots, 0)=0, \quad \text { a.e. } s \in[0,1]
$$

Note that $H\left(t^{*}, s\right)>0, s \in(0,1)$, then we have

$$
f(s, 0, \ldots, 0)=0, \quad \text { a.e. } s \in[0,1]
$$

But on the other hand, since $f\left(t_{0}, 0, \ldots, 0\right) \neq 0, t_{0} \in[0,1]$, we have $f\left(t_{0}, 0, \ldots, 0\right)>0$, by the continuity of $f$, we can find a set $\Omega \subset[0,1]$ satisfying $t_{0} \in \Omega$ and the Lebesgue measure $\mu(\Omega)>0$ such that $f(t, 0, \ldots, 0)>0$ for any $t \in \Omega$. This contradicts to (3.29). Therefore, $x(t)>0$, that is, $x(t)$ is positive solution of (3.1).

Example 3.8. Consider the following fractional boundary value problem:

$$
\begin{gathered}
-\boldsymbol{\Phi}^{5 / 2} x(t)=e^{t}+\frac{1}{10} x(t)+\frac{1}{2} \sin ^{2}\left(\boldsymbol{\Phi}^{1 / 8} x(t)\right)+\frac{1}{3} \cos ^{2}\left(\boldsymbol{\Phi}^{1 / 4} x(t)\right), \quad 0<t<1, \\
\boldsymbol{\Phi}^{1 / 4} x(0)=\boldsymbol{\Phi}^{5 / 4} x(0)=0, \quad \boldsymbol{\Phi}^{1 / 4} x(1)=\frac{1}{4} \boldsymbol{\Phi}^{1 / 4} x\left(\frac{1}{4}\right)+\frac{1}{2} \boldsymbol{\Phi}^{1 / 4} x\left(\frac{3}{4}\right) .
\end{gathered}
$$

Then the BVP (3.30) has a unique positive solution.

Proof. Since

$$
\begin{gathered}
\sum_{j=1}^{m-2} a_{j} \xi_{j}^{\alpha-\mu_{n-1}-1}=\frac{1}{4}\left(\frac{1}{4}\right)^{5 / 4}+\frac{1}{2}\left(\frac{3}{4}\right)^{5 / 4}=0.39316<1 \\
\eta=\frac{1}{\Gamma\left(\alpha-\mu_{n-1}\right)}\left(1+\frac{\sum_{j=1}^{m-2} a_{j}}{1-\sum_{j=1}^{m-2} a_{j} \xi_{j}^{\alpha-\mu_{n-1}-1}}\right)=0.10964 .
\end{gathered}
$$


Thus,

$$
(n \eta)^{-1}=3.0405
$$

Let $K=15$ and $\phi(x)=15 x$, and take

$$
f\left(t, x_{1}, x_{2}, x_{3}\right)=e^{t}+\frac{1}{10} x_{1}+\frac{1}{2} \sin ^{2} x_{2}+\frac{1}{3} \cos ^{2} x_{3}, \quad\left(t, x_{1}, x_{2}, x_{3}\right) \in[0,1] \times[0,+\infty)^{3} .
$$

Then, for any $x_{1} \geq y_{1}, x_{2} \geq y_{2}, x_{3} \geq y_{3}$,

$$
\begin{aligned}
\left|f\left(t, x_{1}, x_{2}, x_{3}\right)-f\left(t, y_{1}, y_{2}, y_{3}\right)\right|= & \left|\frac{x_{1}-y_{1}}{10}+\frac{\sin ^{2} x_{2}-\sin ^{2} y_{2}}{2}+\frac{\cos ^{2} x_{3}-\cos ^{2} y_{3}}{3}\right| \\
\leq & \frac{x_{1}-y_{1}}{10}+\frac{x_{2}-y_{2}}{2}+\frac{x_{3}-y_{3}}{3} \\
= & \frac{2}{75} \times 15 \times \frac{1}{4}\left(x_{1}-y_{1}\right)+\frac{1}{10} \times 15 \times \frac{1}{3}\left(x_{2}-y_{3}\right) \\
& +\frac{4}{9} \times 15 \times \frac{1}{20}\left(x_{3}-y_{3}\right) \\
= & \frac{2}{75} \phi\left(\frac{1}{4}\left(x_{1}-y_{1}\right)\right)+\frac{1}{10} \phi\left(\frac{1}{3}\left(x_{2}-y_{3}\right)\right) \\
& +\frac{4}{9} \phi\left(\frac{1}{20}\left(x_{3}-y_{3}\right)\right) \\
= & \sum_{i=1}^{3} \rho_{i} \phi\left(\theta_{i}\left(x_{i}-y_{i}\right)\right),
\end{aligned}
$$

where

$$
\rho_{1}=\frac{2}{75}, \quad \rho_{2}=\frac{1}{10}, \quad \rho_{3}=\frac{4}{9}, \quad \theta_{1}=\frac{1}{4}, \quad \theta_{2}=\frac{1}{3}, \quad \theta_{3}=\frac{1}{20} .
$$

Thus, $\phi \in \mathbb{B}$ and all of the conditions of Theorem 3.6 are satisfied.

On the other hand, $f(0,0, \ldots, 0)=4 / 3 \neq 0$, by Theorems 3.6 and 3.7, the BVP (19) has a unique positive solution.

Remark 3.9. In Example 3.8, $\beta(x)=\phi(x) / x=K=15$ which does not possess property $\beta$ : $[0,+\infty) \rightarrow[0,1)$ and

$$
\beta\left(t_{n}\right) \longrightarrow 1 \text { implies } t_{n} \longrightarrow 0
$$

Thus, the unique positive solution of BVP (3.30) cannot be obtained via Theorem 1.2, but we obtain the unique positive solution of BVP (3.30) by using the generalized fixed point Theorem 2.2, which implies that Theorem 2.2 is an essential promotion of Theorem 1.2. 


\section{Acknowledgment}

This work is supported by the Natural Sciences of Education and Science Office Bureau of Sichuan Province of China under Grant no. 2010JY0J41,11ZB152.

\section{References}

[1] M. A. Geraghty, "On contractive mappings," Proceedings of the American Mathematical Society, vol. 40, pp. 604-608, 1973.

[2] A. Amini-Harandi and H. Emami, "A fixed point theorem for contraction type maps in partially ordered metric spaces and application to ordinary differential equations," Nonlinear Analysis, vol. 72, no. 5, pp. 2238-2242, 2010.

[3] I. J. Cabrera, J. Harjani, and K. B. Sadarangani, “Existence and uniqueness of positive solutions for a singular fractional three-point boundary value problem," Abstract and Applied Analysis, vol. 2012, Article ID 803417, 18 pages, 2012.

[4] B. Ahmad, A. Alsaedi, and B. S. Alghamdi, "Analytic approximation of solutions of the forced Duffing equation with integral boundary conditions," Nonlinear Analysis: Real World Applications, vol. 9, no. 4, pp. 1727-1740, 2008.

[5] J. Harjani and K. Sadarangani, "Fixed point theorems for weakly contractive mappings in partially ordered sets," Nonlinear Analysis, vol. 71, no. 7-8, pp. 3403-3410, 2009.

[6] Y. Wang, L. Liu, and Y. Wu, "Positive solutions for a class of fractional boundary value problem with changing sign nonlinearity," Nonlinear Analysis, vol. 74, no. 17, pp. 6434-6441, 2011.

[7] X. Zhang and Y. Han, "Existence and uniqueness of positive solutions for higher order nonlocal fractional differential equations," Applied Mathematics Letters, vol. 25, no. 3, pp. 555-560, 2012.

[8] X. Zhang, L. Liu, and Y. Wu, "The eigenvalue problem for a singular higher order fractional differential equation involving fractional derivatives," Applied Mathematics and Computation, vol. 218, no. 17, pp. 8526-8536, 2012.

[9] X. Zhang, L. Liu, B. Wiwatanapataphee, and Y. Wu, "Positive solutions of eigenvalue problems for a class of fractional differential equations with derivatives," Abstract and Applied Analysis, vol. 2012, Article ID 512127, 16 pages, 2012.

[10] X. Zhang, L. Liu, and Y. Wu, "Multiple positive solutions of a singular fractional differential equation with negatively perturbed term," Mathematical and Computer Modelling, vol. 55, no. 3-4, pp. 1263-1274, 2012.

[11] Y. Wang, L. Liu, and Y. Wu, "Positive solutions of a fractional boundary value problem with changing sign nonlinearity," Abstract and Applied Analysis, vol. 2012, Article ID 149849, 12 pages, 2012.

[12] M. Jia, X. Liu, and X. Gu, "Uniqueness and asymptotic behavior of positive solutions for a fractionalorder integral boundary value problem," Abstract and Applied Analysis, vol. 2012, 21 pages, 2012.

[13] I. Podlubny, Fractional Differential Equations, vol. 198 of Mathematics in Science and Engineering, Academic Press, San Diego, Calif, USA, 1999.

[14] I. Podlubny, "Geometric and physical interpretation of fractional integration and fractional differentiation," Fractional Calculus \& Applied Analysis, vol. 5, no. 4, pp. 367-386, 2002.

[15] X. Zhang, L. Liu, and Y. Wu, "Existence results for multiple positive solutions of nonlinear higher order perturbed fractional differential equations with derivatives," Applied Mathematics and Computation, vol. 219, no. 4, pp. 1420-1433, 2012. 


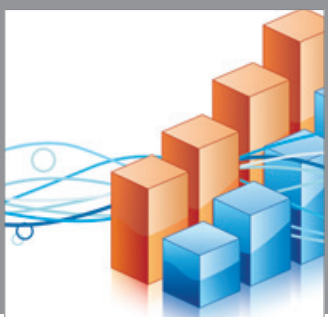

Advances in

Operations Research

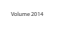

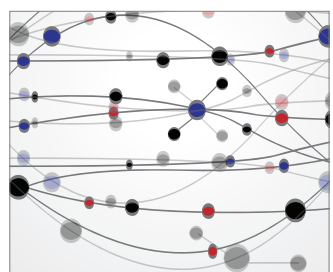

\section{The Scientific} World Journal
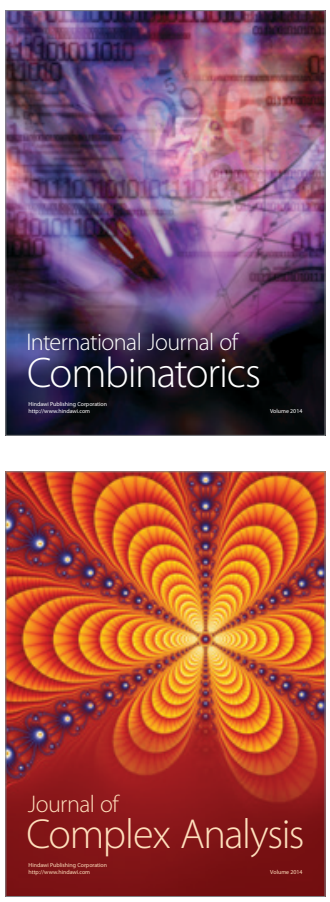

International Journal of

Mathematics and

Mathematical

Sciences
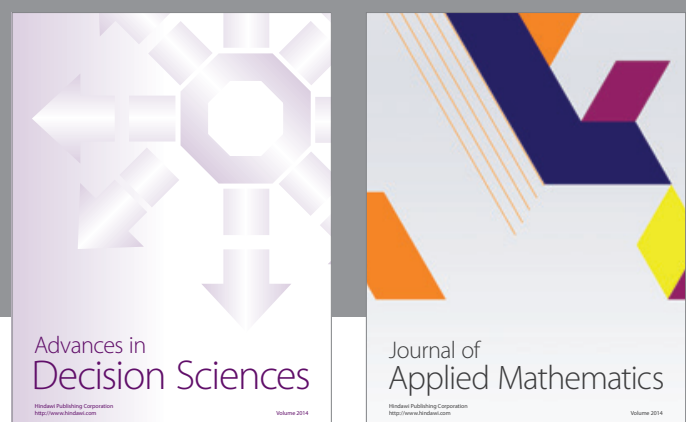

Journal of

Applied Mathematics
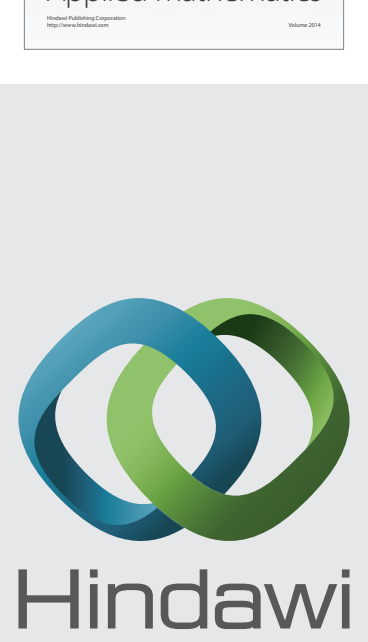

Submit your manuscripts at http://www.hindawi.com
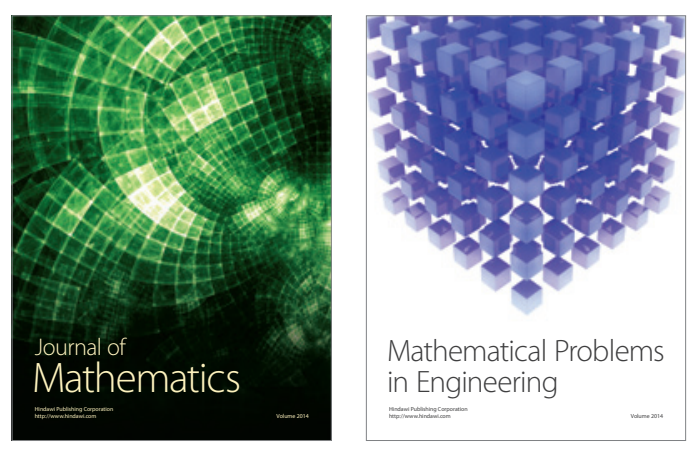

Mathematical Problems in Engineering
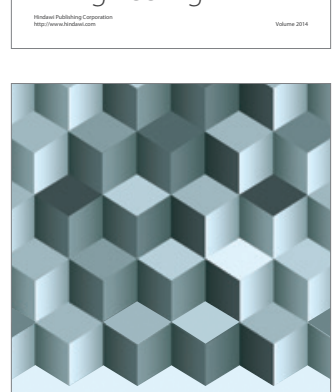

Journal of

Function Spaces
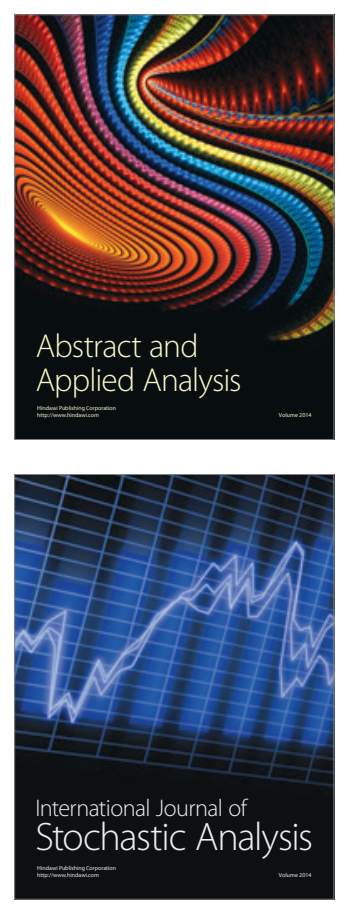

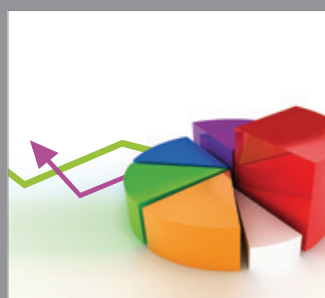

ournal of

Probability and Statistics

Promensencen
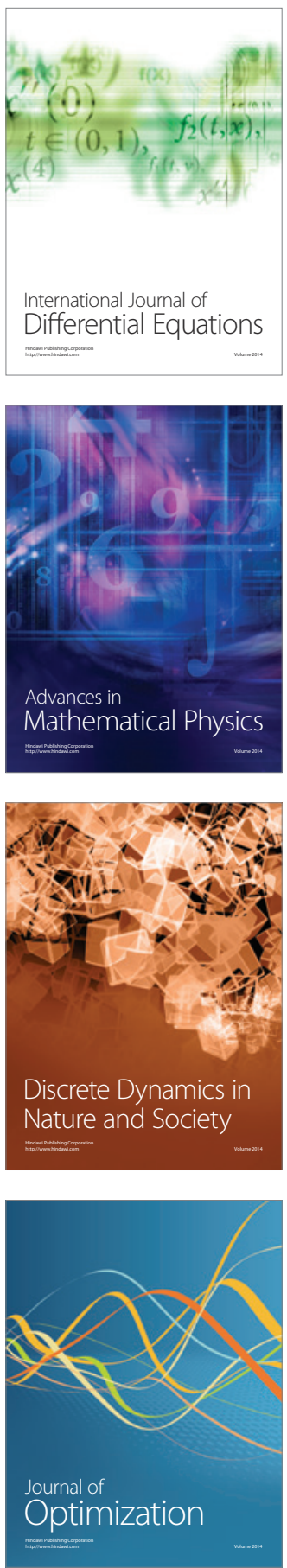